\title{
THE ROLE OF THE MEAN CURVATURE IN THE IMMERSION THEORY OF SURFACES*
}

\author{
BY \\ H. W. ALEXANDER
}

1. Introduction. In this paper it is proposed to deal with the immersion theory of surfaces from a point of view somewhat different from the classical. Part I, sections 3-6, will be devoted to an exposition of the role of the mean curvature in the immersion theory. In the general case, expressions will be obtained for the second fundamental tensor $\Omega_{\alpha \beta}$ in terms of the mean curvature, the first fundamental tensor and their derivatives; and necessary and sufficient conditions will be derived in order that a function $K_{m}\left(u^{\alpha}\right)$ may constitute the mean curvature of a surface with given linear element. Given a function $K_{m}\left(u^{\alpha}\right)$ satisfying these conditions, the surface will be determined to within rigid motions in space, except in certain particular cases, which will be given careful treatment.

The dependence of the immersion on the mean curvature is considered in two papers by W. C. Graustein [1], in the first of which ample references to the literature are given. The expression for $\Omega_{\alpha \beta}$ in terms of the mean curvature, and the differential equations governing the mean curvature, do not appear to have been obtained before.

Part II, sections 7-11, will deal with an important type of singularity of the immersion, referred to as the edge of regression, which is the envelope of both sets of asymptotic lines, and for which the mean curvature is infinite. The geometrical properties of the edge, both local and in the large, as well as its analytical structure, will be studied in these sections.

\section{PART I}

2. Notation and definition. In Part I we shall be dealing purely with the local geometry of a surface; that is, with a region $R$ of the surface which is homeomorphic to the interior of a circle of the Euclidean plane. The coordinates $u^{\alpha}, \alpha=1,2$, of a point of $R$ are defined to be the Cartesian coordinates of its image under the homeomorphism, and the homeomorphism defines a coordinate system over $R$, denoted by $\left[u^{\alpha}\right]$. The functions we shall consider are assumed to possess a sufficient number of derivatives so that the quantities defined shall exist.

\footnotetext{
* Presented to the Society, in part on September 9, 1937, and in part on December 28, 1939; received by the editors July 12, 1939, and, in revised form, November 18, 1939.
} 
The region $R$ belongs to an immersed surface if to each point of $R$ there corresponds an ordered triad of numbers $x^{i}, i=1,2,3,{ }^{*}$ called its space coordinates, such that the sum of the squares of the Jacobians of the $x$ 's with respect to the $u$ 's is not identically zero.

The region $R$ belongs to an intrinsic surface if to each coordinate system there corresponds an ordered triad of functions $g_{11}\left(u^{\alpha}\right), g_{12}\left(u^{\alpha}\right)=g_{21}\left(u^{\alpha}\right)$, $g_{22}\left(u^{\alpha}\right)$, called the components of its first fundamental tensor, such that $g=\left|g_{\alpha \beta}\right| \neq 0, g_{\alpha \beta} d u^{\alpha} d u^{\beta}$ is positive definite, and $g_{\alpha \beta}$ undergoes the transformation

$$
\bar{g}_{\alpha \beta}=g_{\gamma \delta} \frac{\partial u^{\gamma}}{\partial \bar{u}^{\alpha}} \frac{\partial u^{\delta}}{\partial \bar{u}^{\beta}}
$$

when the coordinate system $\left[u^{\alpha}\right]$ is transformed into the coordinate system $\left[\bar{u}^{\alpha}\right]$. The surface is regular at a point $P$ if there exists a coordinate system for which $g \neq 0$ at $P$. We shall deal only with such surfaces.

From an immersed surface we can obtain an intrinsic surface by defining the fundamental tensor

$$
g_{\alpha \beta}=\frac{\partial x^{i}}{\partial u^{\alpha}} \cdot \frac{\partial x^{i}}{\partial u^{\beta}}
$$

conversely, given an intrinsic surface by means of the functions $g_{\alpha \beta}\left(u^{\gamma}\right)$, we may be able to integrate (2.1) to obtain an immersed surface.

If $\varepsilon_{i j k}$ denotes a factor which is zero unless $i, j, k$ are different, and 1 or -1 according as $i j k$ are in cyclic or anticyclic order, and if $\varepsilon^{\alpha \beta}$ is the skew symmetric tensor whose components are [2]

$$
\varepsilon^{11}=\varepsilon^{22}=0, \quad \varepsilon^{12}=-\varepsilon^{21}=g^{-1 / 2},
$$

then the second fundamental tensor is given by

$$
\Omega_{\alpha \beta}=\varepsilon_{i j k} \varepsilon^{\gamma \delta} \frac{\partial x^{i}}{\partial u^{\gamma}} \frac{\partial x^{j}}{\partial u^{\delta}} \frac{\partial^{2} x^{k}}{\partial u^{\alpha} \partial u^{\beta}}
$$

and the mean curvature by

$$
K_{m}=g^{\alpha \beta} \Omega_{\alpha \beta} .
$$

The quantities $\Omega_{\alpha \beta}$ satisfy the equations of Codazzi

$$
\Omega_{\alpha \beta, \gamma}-\Omega_{\alpha \gamma, \beta}=0,
$$

where the comma indicates covariant differentiation, and satisfy the equations of Gauss $1,2,3$.

* We shall employ Greek indices to denote the range 1, 2, and Latin indices to denote the range 


$$
K g=\Omega,
$$

where $\Omega$ is the determinant of $\Omega_{\alpha \beta}$, and $K$ is the Gaussian curvature, which is expressible in terms of the $g_{\alpha \beta}$ and their derivatives.

In the classical treatment, the immersion theory of surfaces is broken down into two stages: first, the discovery of a tensor $\Omega_{\alpha \beta}$ satisfying the equations of Gauss and Codazzi; and, second, the integration of certain equations of the Riccati type to obtain the space coordinates of the surface. The theorem is proved that if an intrinsic surface is initially given, and if there exist functions $\Omega_{\alpha \beta}\left(u^{\gamma}\right)$ satisfying the equations of Gauss and Codazzi, then there exists an immersion of the surface, unique to within rigid motions in space, for which $\Omega_{\alpha \beta}\left(u^{\gamma}\right)$ is the second fundamental tensor. In the present paper it is proposed to introduce a stage prior to the two mentioned: namely, to discover a single function $K_{m}$ (the mean curvature) satisfying, in general, two partial differential equations of the third order. The tensor $\Omega_{\alpha \beta}$ may then be obtained without integration.

We shall assume that the region $R$ is free from umbilics. That is, if $\rho_{1}$ and $\rho_{2}$ are the principal radii of curvature, $\rho_{1} \neq \rho_{2}$ over $R$. Let us choose $\rho_{2}$ to be the larger of the two radii, and define

$$
M=\frac{K_{m}}{2}=(1 / 2)\left(\frac{1}{\rho_{1}}+\frac{1}{\rho_{2}}\right), \quad N=\left(M^{2}-K\right)^{1 / 2}=(1 / 2)\left(\frac{1}{\rho_{1}}-\frac{1}{\rho_{2}}\right) .
$$

As a notational convenience we introduce the idea of the "bisector tensor" of a real symmetric tensor. The symmetric tensor $a_{\alpha \beta}^{*}$ is the bisector tensor of the symmetric tensor $a_{\alpha \beta}$ if $a_{\alpha \beta}^{*} d u^{\alpha} d u^{\beta}$ is the Jacobian of $a_{\alpha \beta} d u^{\alpha} d u^{\beta}$ and $g_{\alpha \beta} d u^{\alpha} d u^{\beta}$ divided by $4 g^{1 / 2}$. It may be shown that

$$
a_{\alpha \beta}^{*}=(1 / 2)\left(\varepsilon_{\cdot \alpha}^{\delta} a_{\delta \beta}+\varepsilon_{\cdot \beta}^{\delta} a_{\delta \alpha}\right),
$$

where $\varepsilon_{. \alpha}^{\delta}=g_{\gamma \alpha} \varepsilon^{\delta \gamma}$. By definition the directions $a_{\alpha \beta}^{*} d u^{\alpha} d u^{\beta}=0$ bisect the angles between the directions $a_{\alpha \beta} d u^{\alpha} d u^{\beta}=0$. Thus the equations $\Omega_{\alpha \beta} d u^{\alpha} d u^{\beta}=0$ and $\Omega_{\alpha \beta}^{*} d u^{\alpha} d u^{\beta}=0$ define, respectively, the asymptotic and principal directions.

The bisector tensor satisfies the identity

$$
a^{*}=\left|a_{\alpha \beta}^{*}\right|=a-(g / 4)\left(g^{\alpha \beta} a_{\alpha \beta}\right)^{2} .
$$

The following three conditions, $\mathrm{A}, \mathrm{B}$ and $\mathrm{C}$, are equivalent to each other:

$$
\mathrm{A}: a^{*}=0
$$
$\mathrm{B}: \frac{a_{11}}{g_{11}}=\frac{a_{12}}{g_{12}}=\frac{a_{22}}{g_{22}} ;$
$\mathrm{C}: a_{\alpha \beta}^{*}=0$. 
3. General expressions for $\Omega_{\alpha \beta}$. In $\$ \S 3$ and 4 we shall assume we are given an immersed surface, and we shall obtain necessary conditions on the mean curvature, and on an invariant $\phi$ to be defined.

THEOREM 3.1. Let $A\left(u^{\alpha}\right)$ be any real non-constant scalar (that is, $\left.\Delta_{1} A \neq 0\right)$, $\dagger$ and let $b_{\alpha \beta}$ be the tensor $\left(2 N A_{, \alpha} A_{, \beta}\right) / \Delta_{1} A$. Let $\phi$ be twice the angle from the vector $A_{, \alpha}$ to the line of curvature whose principal radius is $\rho_{1}$. Then

$$
\Omega_{\alpha \beta}=\cos \phi\left(b_{\alpha \beta}-N g_{\alpha \beta}\right)+\sin \phi b_{\alpha \beta}^{*}+M g_{\alpha \beta},
$$

where $b_{\alpha \beta}^{*}$ is the bisector tensor of $b_{\alpha \beta}$.

Proof. Use the coordinate system consisting of the lines $A=$ const. and their orthogonal trajectories, with $u^{1}=A$. In this system equations (3.1) reduce to

(3.2) $\Omega_{11}=g_{11}(N \cos \phi+M), \Omega_{12}=g^{1 / 2} N \sin \phi, \Omega_{22}=g_{22}(-N \cos \phi+M)$.

If $\theta$ is the angle between the direction $d u^{\alpha}$ and the line curvature whose principal radius is $\rho_{1}$, then from Euler's theorem the normal curvature corresponding to the direction $d u^{\alpha}$ is given by

$$
\frac{\Omega_{\alpha \beta} d u^{\alpha} d u^{\beta}}{g_{\alpha \beta} d u^{\alpha} d u^{\beta}}=N \cos 2 \theta+M .
$$

Using (3.3) with $d u^{2}=0,2 \theta=\phi$, we obtain the expression for $\Omega_{11}$ given in (3.2). Using (3.3) with $d u^{1}=0$ and $2 \theta=\phi+\pi$, we obtain $\Omega_{22}$. Finally, to obtain $\Omega_{12}$ we use

$$
\Omega_{12}^{2}=\Omega_{11} \Omega_{22}-K g=g N^{2} \sin ^{2} \phi .
$$

Thus equation (3.2) is verified.

TheOREM 3.2. The invariant $\phi$ satisfies the differential equation

$$
\begin{aligned}
\phi_{, \alpha}=\frac{1}{N \Delta_{1} A}\left[2 A_{, \gamma} \Delta_{1}(M, A)-M_{, \gamma} \Delta_{1} A\right] \cdot\left[\boldsymbol{\varepsilon}_{\cdot \alpha}^{\gamma} \cos \phi-\delta_{\alpha}^{\gamma} \sin \phi\right] \\
-\frac{2 \boldsymbol{\varepsilon}^{\beta \gamma} A_{, \beta} A_{, \gamma \alpha}}{\Delta_{1} A}-\frac{\boldsymbol{\varepsilon}_{\cdot \alpha}^{\gamma} N_{, \gamma}}{N},
\end{aligned}
$$

where $\delta_{\alpha}^{\gamma}$ is the Kronecker delta.

By using the coordinate system of the proof of Theorem 3.1, it is readily verified that equations (3.4) are equivalent to the Codazzi equations (2.2), where $\Omega_{\alpha \beta}$ is given by (3.1).

$\dagger$ Throughout the paper the signs $=$ and $\neq$ will be assumed to hold at every point of the region $R$. 
4. Necessary conditions on the mean curvature. From this point on we shall specialize the invariant $A$ to equal either $M$ or $K$. If $M$ is not constant, we put $A=M$; if $M$ is constant, but $K$ is not, we put $A=K$. It is easily proved that if both $M$ and $K$ are constant, the surface reduces to a plane, a sphere or a circular cylinder.

We consider first the case $\Delta_{1} M \neq 0$. Then we may take $A=M$, and (3.4) reduces to

$$
\phi, \alpha=\frac{M_{, \gamma}}{N}\left(\varepsilon_{\cdot \alpha}^{\gamma} \cos \phi-\delta_{\alpha}^{\gamma} \sin \phi\right)-\frac{2 \varepsilon^{\beta \gamma} M_{, \beta} M_{, \gamma \alpha}}{\Delta_{1} M}-\varepsilon_{\cdot \alpha}^{\gamma} \frac{N_{, \gamma}}{N} .
$$

TheOrem 4.1. The invariants $M$ and $\phi$ satisfy the equation

$$
R \cos \phi+S \sin \phi+T=0,
$$

where†

$$
\begin{aligned}
& R=(1 / N)\left[\Delta_{2} M-\Delta_{1}\left(M, \log \Delta_{1} M / N^{2}\right)\right] \\
& S=(-1 / N) \Theta\left(M, \log \Delta_{1} M / N^{2}\right), \\
& T=\Delta_{2} \log N-\Delta_{1} M / N^{2}-2 K .
\end{aligned}
$$

Equation (4.2) is the condition of integrability of equations (4.1). That this is the case may be verified by using the coordinate system of the proof of Theorem 3.1 with $A=M$.

The invariant $T$ of (4.3) may also be expressed in the form

$$
T=\frac{-M}{N^{2}} g^{\alpha \beta} a_{\alpha \beta}-\frac{\Delta_{2} K}{2 N^{2}}-\frac{\Delta_{1} K}{2 N^{4}}-2 K,
$$

where

$$
a_{\alpha \beta}=-M_{, \alpha \beta}+(1 / N)\left(M_{, \alpha} N_{, \beta}+M_{, \beta} N_{, \alpha}\right) .
$$

This expression is obtained from those of (4.3) by expanding $\Delta_{2} \log N$ $=(1 / 2) \Delta_{2} \log \left(M^{2}-K\right)$.

If $\Omega^{* \alpha \beta}$ is the cofactor of $\Omega_{\alpha \beta}^{*}$ in the determinant $\left|\Omega_{\alpha \beta}^{*}\right|$ divided by $\Omega^{*}=-g N^{2}$, and if $P$ and $Q$ are given by $P=-\Omega^{* \alpha \beta} a_{\alpha \beta}, Q=-\Omega^{*_{\alpha \beta} \beta} a_{\alpha \beta}^{*}$, where $a_{\alpha \beta}$ is given by (4.5), then $P$ and $Q$ satisfy the identities

$$
\begin{gathered}
P=-S \cos \phi+R \sin \phi, \quad Q=-R \cos \phi-S \sin \phi, \\
P^{2}+Q^{2}=R^{2}+S^{2}=-4 a^{*} / g N^{2} .
\end{gathered}
$$

The proof follows readily when the coordinate system of Theorem 3.1, with $A=M$, is employed.

$\dagger \Theta(A, B)$ is defined by $\Theta(A, B)=\varepsilon^{\alpha \beta} A_{, \alpha} B, \beta$. 
From (4.2) and (4.6) we have $Q=T$; and hence, using (4.6) it follows that $P$ and $Q$ are both expressible in terms of $M$ and $g_{\alpha \beta}$ and their derivatives:

$$
P=\epsilon\left(R^{2}+S^{2}-T^{2}\right)^{1 / 2}, \quad Q=T, \quad \epsilon= \pm 1 .
$$

Throughout the remainder of this section, and also in $\$ 5$, we shall be concerned with the case in which $R$ and $S$ are not both zero. Since $R$ and $S$ are real functions, this condition is equivalent to $R^{2}+S^{2} \neq 0$, or $P^{2}+Q^{2} \neq 0$, or $a^{*} \neq 0$. Then we may solve equations (4.6) for $\sin \phi$ and $\cos \phi$ :

$$
\sin \phi=\frac{R P-S Q}{P^{2}+Q^{2}}, \quad \cos \phi=\frac{-S P-R Q}{P^{2}+Q^{2}} .
$$

If we substitute the values for $P$ and $Q$ from (4.7), we obtain

$$
\begin{aligned}
& \sin \phi=\frac{-S T+\epsilon R\left(R^{2}+S^{2}-T^{2}\right)^{1 / 2}}{R^{2}+S^{2}}, \\
& \cos \phi=\frac{-R T-\epsilon S\left(R^{2}+S^{2}-T^{2}\right)^{1 / 2}}{R^{2}+S^{2}} ;
\end{aligned}
$$

these expressions could be obtained more directly by solving equation (4.2) for $\sin \phi$ and $\cos \phi$. When the expressions (4.8) are substituted in (3.1) with $A=M$, we obtain the result stated in the following theorem.

THEOREM 4.2. If $R$ and $S$ are not both zero, the second fundamental tensor satisfies the identity

$$
\Omega_{\alpha \beta}=U a_{\alpha \beta}+\epsilon V a_{\alpha \beta}^{*}+W g_{\alpha \beta}, \quad \epsilon= \pm 1
$$

where

$$
\begin{gathered}
a_{\alpha \beta}=-M_{, \alpha \beta}+(1 / N)\left(M_{, \alpha} N_{, \beta}+M_{, \beta} N_{, \alpha}\right), \\
U=\frac{-2 T}{R^{2}+S^{2}}, \quad V=\frac{2\left(R^{2}+S^{2}-T^{2}\right)^{1 / 2}}{R^{2}+S^{2}}, W=M-\frac{U}{2} g^{\alpha \beta} a_{\alpha \beta},
\end{gathered}
$$

and $R, S$ and $T$ have the values given in (4.3).

We may use the coordinate system of Theorem 3.1, with $A=M$, and equations (3.2) for the components of $\Omega_{\alpha \beta}$. The theorem then follows readily with the aid of (4.8).

When we substitute the expressions (4.9) in the equations (2.2) of Codazzi, we obtain two third order differential equations on the mean curvature which may be written

$$
\varepsilon^{\beta \gamma}\left(U a_{\alpha \beta}+\epsilon V a_{\alpha \beta}^{*}+W g_{\alpha \beta}\right)_{, \gamma}=0,
$$


where $\epsilon$ has the same value as in (4.9). These are thus necessary conditions on the mean curvature in the case $R^{2}+S^{2} \neq 0$.

5. Sufficiency of the conditions (4.11). In this section the problem of immersing a given intrinsic surface will be reduced, in the general case, to finding a function $M\left(u^{\alpha}\right)$ satisfying equations (4.11). Exceptional cases will be treated in $\$ 6$.

TheOREM 5.1. If $M\left(u^{\alpha}\right)$ is any real solution of equations (4.11), then there exists an immersed surface, determined uniquely to within rigid motions in space, for which $2 M\left(u^{\alpha}\right)$ is the mean curvature and $g_{\alpha \beta}$ the fundamental tensor.

Proof. It is readily verified that since (4.11) is satisfied, $\Omega_{\alpha \beta}$ as defined by (4.9) satisfies the equations of Gauss and Codazzi. Thus the existence of the surface follows from classical theory, and its second fundamental tensor is given by (4.9). Moreover, $g^{\alpha \beta} \Omega_{\alpha \beta}=2 M$, so that $2 M\left(u^{\alpha}\right)$ is actually the mean curvature.

The ambiguity of sign in the expression for $\Omega_{\alpha \beta}$ in (4.9) has the following consequences. If $M\left(u^{\alpha}\right)$ is a solution of (4.11) with $\epsilon=1$, then equations (4.9) with $\epsilon=1$ yield a tensor $\Omega_{\alpha \beta}$ satisfying the equations of Gauss and Codazzi; a similar statement holds for $\epsilon=-1$. If it happens, however, that $M\left(u^{\alpha}\right)$ satisfies the pair of equations

$$
\varepsilon^{\beta \gamma}\left(V a_{\alpha \beta}^{*}\right)_{, \gamma}=0
$$

in addition to (4.11), then the expression (4.9) with $\epsilon$ equal to either 1 or -1 will satisfy the equations of Gauss and Codazzi. In this case, from a single function $M\left(u^{\alpha}\right)$ we would be led to two surfaces, intrinsically identical, but having different immersions [3]. We remark that equations (4.11) together with (5.1) would lead to certain necessary conditions on the intrinsic geometry of the surface; these will not be investigated here.

6. The case $R=S=0$. In this case it follows from (4.2) that $T=0$; and from the identities (4.6) we have $P=Q=a^{*}=0$.

THEOREM 6.1. The condition $P=-\Omega^{* \alpha \beta} a_{\alpha \beta}=0$ is necessary and sufficient that the surface be isometric, that is, that the lines of curvature form an isometric system.

The proof consists in taking the lines of curvature as parametric, and recalling that the condition that the parametric lines be isometric [4] is $\partial^{2} \log \left(g_{11} / g_{22}\right) / \partial u^{1} \partial u^{2}=0$. This condition may be reduced to $P=0$ by making use of the Codazzi equations together with the relations

$$
\Omega_{11}=g_{11}(M+N), \quad \Omega_{22}=g_{22}(M-N) .
$$


Since $a^{*}=0$, from (2.3) we have $a_{11} / g_{11}=a_{12} / g_{12}=a_{22} / g_{22}$. If we write these equations in the form $a_{\alpha \beta}=\rho g_{\alpha \beta}$, and multiply through by $g^{\alpha \beta}$, we obtain $\rho=(1 / 2) g^{\alpha \beta} a_{\alpha \beta}$. But since $T=0$, from (4.4) we have

$$
g^{\alpha \beta} a_{\alpha \beta}=-\left(1 / 2 M N^{2}\right)\left(N^{2} \Delta_{2} K+\Delta_{1} K+4 N^{4} K\right) .
$$

Thus

$$
a_{\alpha \beta}=-\left(g_{\alpha \beta} / 4 M N^{2}\right)\left(N^{2} \Delta_{2} K+\Delta_{1} K+4 N^{4} K\right)
$$

or

$$
\begin{aligned}
M_{, \alpha \beta}= & (1 / N)\left(M_{, \alpha} N_{, \beta}+M_{, \beta} N_{, \alpha}\right) \\
& +\left(g_{\alpha \beta} / 4 M N^{2}\right)\left(N^{2} \Delta_{2} K+\Delta_{1} K+4 N^{4} K\right) .
\end{aligned}
$$

Conversely, it is readily shown that equations (6.1) imply $R=0, S=0$ and $T=0$.

Equations (6.1) lead to certain necessary conditions on the intrinsic geometry, which we may obtain in the following way. Let us write (6.1) in the form

$$
M_{, \alpha \beta}=f_{\alpha \beta}\left(M_{, \alpha}, M, g_{\alpha \beta}\right),
$$

where $f_{\alpha \beta}$ involves the derivatives of $g_{\alpha \beta}$; this will be understood also in the functions $F_{\alpha}$ and $\Phi$ below.

To obtain integrability conditions on (6.2), we make use of the identity

$$
\varepsilon^{\beta \gamma} M_{, \alpha \beta \gamma}=-K_{\varepsilon_{\cdot \alpha}^{\beta}}^{\beta} M_{, \beta} .
$$

This is derived from the identity [5]

$$
\lambda_{\alpha, \beta \gamma}-\lambda_{\alpha, \gamma \beta}=\lambda_{\delta} \delta \delta^{\delta \mu} R_{\mu \alpha \beta \gamma},
$$

in view of the fact that for a two dimensional surface all the components of $R_{\lambda \alpha \beta \gamma}$ vanish except $R_{1212}=R_{2121}=-R_{1221}=-R_{2112}=g K$.

When we differentiate covariantly the expressions (6.2) for $M_{, \alpha \beta}$, substitute in (6.3), eliminate $M_{, \alpha \beta}$ and solve for $M_{, \alpha}$, we get an equation of the form

$$
M_{, \alpha}=F_{\alpha}\left(M, g_{\alpha \beta}\right) .
$$

We now develop the integrability condition of (6.4), eliminate $M_{, \alpha}$ and solve for $M$, obtaining

$$
M=\Phi\left(g_{\alpha \beta}\right) .
$$

Thus we may obtain a set of necessary conditions on $g_{\alpha \beta}$ by substituting $M$ from (6.5) in (6.2):

$$
\Phi_{, \alpha \beta}=f_{\alpha \beta}\left(\Phi_{, \alpha}, \Phi, g_{\alpha \beta}\right) .
$$


Conversely, if $g_{\alpha \beta}\left(u^{\gamma}\right)$ is such that (6.6) are identically satisfied, then $M$ defined by (6.5) will be a solution of (6.2).

Certain details have been obtained for the case (of greatest generality) when $\Delta_{1}\left(\Delta_{1} K\right) \neq 0, \Theta\left(K, \Delta_{1} K\right) \neq 0$. In this case it turns out that (6.4) can be written in the form

$$
M_{, \alpha}=a K_{, \alpha}+b\left(\Delta_{1} K\right)_{, \alpha}
$$

where $b$ satisfies a cubic equation $b^{3}+A b^{2}+B b+C=0$, and $a$ is given by $a=(D b+E) /(F b+G)$. The coefficients $A, B, C, D, E, F$ and $G$ are polynomials in $M$ with coefficients formed from the differential parameters of $K$. The author is not able to give any indication of the form of (6.5).

Suppose that (6.6) are satisfied, and that $M\left(u^{\alpha}\right)$ is a solution of (6.1), such that $\Delta_{1} M \neq 0$. Then equations (4.1):

$$
\phi_{, \alpha}=\frac{M_{, \gamma}}{N}\left(\varepsilon_{\cdot \alpha}^{\gamma} \cos \phi-\delta_{\alpha}^{\gamma} \sin \phi\right)-\frac{2 \varepsilon^{\beta \gamma} M_{, \beta} M_{, \gamma \alpha}}{\Delta_{1} M}-\varepsilon_{\cdot \alpha}^{\gamma} \frac{N_{, \gamma}}{N}
$$

are completely integrable. Thus we have an infinity of solutions $\phi\left(u^{\alpha}, a\right)$, where $a$ is an arbitrary parameter. Consider the functions $\Omega_{\alpha \beta}\left(u^{\gamma}, a\right)$ defined by (3.1) with $A=M$ :

$$
\Omega_{\alpha \beta}=\cos \phi\left(b_{\alpha \beta}-N g_{\alpha \beta}\right)+\sin \phi b_{\alpha \beta}^{*}+M g_{\alpha \beta}, \quad b_{\alpha \beta}=2 N M_{, \alpha} M_{, \beta} / \Delta_{1} M .
$$

It is easily verified that the tensor $\Omega_{\alpha \beta}$ so defined satisfies the equation of Gauss; it also satisfies the equations of Codazzi, since $\phi$ satisfies (4.1). Moreover, it may be shown, as in Theorem 5.1, that $2 M\left(u^{\gamma}, a\right)$ is actually $g^{\alpha \beta} \Omega_{\alpha \beta}\left(u^{\gamma}, a\right)$. Hence we have

THEOREM 6.2. If $M\left(u^{\alpha}\right)$ is any solution of equations (6.1) such that $\Delta_{1} M \neq 0$, then there exist [6] $\infty^{1}$ surfaces with the intrinsic geometry $g_{\alpha \beta}\left(u^{\gamma}\right)$ and the mean curvature $2 M\left(u^{\alpha}\right)$.

The case $\Delta_{1} M=0$, or $M=$ const., automatically satisfies $R^{2}+S^{2}=0$, as is evident from (4.3); but the above discussion is inapplicable, since equations (4.1) are only valid for $\Delta_{1} M \neq 0$. We first consider the case $\Delta_{1} M=0, \Delta_{1} K \neq 0$. Then we may put $A=K$ in equations (3.1) and (3.4). Remembering that $N^{2}=M^{2}-K$, and $M_{, \alpha}=0$, we get

$$
\Omega_{\alpha \beta}=\cos \phi\left(b_{\alpha \beta}-N g_{\alpha \beta}\right)+\sin \phi b_{\alpha \beta}^{*}+M g_{\alpha \beta}, \quad b_{\alpha \beta}=2 N K_{, \alpha} K_{, \beta} / \Delta_{1} K
$$

and

$$
\phi, \alpha=\frac{-2 \varepsilon^{\beta \gamma} K_{, \beta} K_{, \gamma \alpha}}{\Delta_{1} K}+\frac{\varepsilon_{\cdot \alpha}^{\gamma} K_{, \gamma}}{2 N^{2}}
$$


If, as in Theorem 4.1, we develop the integrability conditions of (6.8), we obtain the single equation

$$
N^{2} \Delta_{2} K+\Delta_{1} K+4 N^{4} K=0 .
$$

The method of proof is identical with that used in Theorem 4.1, except that the curves $K=$ const. and their orthogonal trajectories are taken to be parametric.

We can eliminate $M$ entirely from this equation by first writing it in the form

$$
4 K M^{4}+M^{2}\left(\Delta_{2} K-8 K^{2}\right)+4 K^{3}-K \Delta_{2} K+\Delta_{1} K=0 .
$$

When we solve for $M^{2}$ and substitute in $\Delta_{1}\left(M^{2}\right)=0$, we get

$$
\Delta_{1} k=0, \quad k=\frac{8 K^{2}-\Delta_{2} K \pm\left(\Delta_{2}^{2} K-16 K \Delta_{1} K\right)^{1 / 2}}{K} .
$$

In the particular case $M=0,(6.9)$ reduces to

$$
K \Delta_{2} K-\Delta_{1} K-4 K^{3}=0
$$

which is thus a necessary condition on the intrinsic geometry of a minimal surface [7].

Conversely, suppose that we are given an intrinsic surface $g_{\alpha \beta}\left(u^{\gamma}\right)$ satisfying (6.10) with either the + or - sign, say the + sign for definiteness. Then the expression $k$ of (6.10) is a constant. If $k \geqq 0$, then (6.9) is satisfied with $M=(1 / 2) k^{1 / 2}$. Hence equations (6.8) are completely integrable, and we have

$$
\phi=\int\left[\frac{-2 \varepsilon^{\beta \gamma} K_{, \beta} K_{, \gamma \alpha}}{\Delta_{1} K}+\frac{\varepsilon_{\cdot \alpha}^{\gamma} K_{, \gamma}}{2 N^{2}}\right] d u^{\alpha}+C .
$$

There are thus $\infty^{1}$ values of $\phi$ differing by an additive constant. As in the remark preceding Theorem 6.2 we may conclude that $\Omega_{\alpha \beta}\left(u^{\gamma}, C\right)$ as defined by (6.7) satisfies the equations of Gauss and Codazzi, and that $2 M=k^{1 / 2}$ is the corresponding mean curvature. Hence we have

THEOREM 6.3. If $g_{\alpha \beta}\left(u^{\gamma}\right)$ satisfies $\Delta_{1} k=0$ where

$$
k=\frac{8 K^{2}-\Delta_{2} K \pm\left(\Delta_{2}^{2} K-16 K \Delta_{1} K\right)^{1 / 2}}{K},
$$

and if $k \geqq 0$, then there are $\infty^{1}$ surfaces $\Sigma$ with the intrinsic geometry $S$ and the constant mean curvature $k^{1 / 2}$.

It has already been pointed out that if $\Delta_{1} M=\Delta_{1} K=0$, the surface reduces to a plane, a sphere or a circular cylinder. 


\section{PART II}

7. The edge of regression: definition. In view of the significance of the mean curvature in the immersion theory of surfaces, it becomes natural to consider what kind of singularities the function $K_{m}$ may exhibit, and what corresponding singularities will arise on the immersed surface. We shall now consider a certain curve $E$ on a surface along which $K_{m}$ is infinite, and which is the analogue for a general surface of the edge of regression of a developable surface. It will be referred to as the edge of regression; this name is appropriate not only because of the analogy with a developable surface, but also because it has been used to designate the envelope of a congruence of curves in 3-space [8].

In order to provide an adequate foundation for the study of the edge of regression, both local and in the large, we shall first define an immersed surface with boundary, and then state conditions in order that the boundary may constitute an edge of regression. For convenience, we shall speak in terms of analytic functions; but all the properties obtained would continue to hold if the functions involved possessed only a finite number of derivatives.

The region of the Euclidean plane defined by $\left|u^{\alpha}\right|<\epsilon$ will be called a square; the region defined by $\left|u^{1}\right|<\epsilon, 0 \leqq u^{2}<\epsilon$, will be called a half square, and the segment $u^{2}=0,\left|u^{1}\right|<\epsilon$ will be called its bounding edge. The homeomorphic image of a square is of course a region; we shall refer to the homeomorphic image of a half square as a half region, and the image of its bounding edge will be called the bounding edge of the half region.

Consider a two-dimensional manifold together with its boundary. The totality of interior points will be denoted by $A$, and the totality of boundary points by $B$. $A+B$ shall be coverable by a finite number of regions $R_{i}$, $i=1,2, \cdots$, and half regions $\bar{R}_{j}, j=1,2, \cdots$, each homeomorphic to a square $S_{i}$ or a half square $\bar{S}_{j}$ of the Euclidean plane. The regions $R_{i}$ lie on $A$ and the half regions $\bar{R}_{j}$ have their interior part on $A$ and their bounding edge on $B$. The coordinates $u^{\alpha}, \alpha=1,2$, of a point with respect to a particular $R_{i}$ or $\bar{R}_{j}$ to which it belongs are defined to be the Cartesian coordinates of its image under the homemorphism. If a point belongs to more than one of the sets $R_{i}$ or $\bar{R}_{j}$, the transformation from one coordinate system to the other will be assumed to be analytic. To each point of $A+B$ there shall correspond an ordered triad of real numbers $x^{i}, i=1,2,3$, such that the sum of the squares of the Jacobians of the $x$ 's with respect to the $u$ 's is different from zero.

Let $\Sigma$ be an immersed surface and let the curve $E$ be its bounding edge. $E$ will be assumed to possess a unique tangent at every point. The plane 
normal to $E$ through an arbitrary point $P$ of $E$ will cut the surface in a curve $C$ bounded by $P$. We define as follows a coordinate system $[s, t]$ for a certain half region $\bar{R}$ whose bounding edge lies on $E: s$ is the arc length along $E$ from a fixed point $O$ to $P$, and $t$ is the arc length along $C$ measured from $P$. The curve $E$ will be called an analytic edge of regression if it satisfies the following three conditions:

CoNDition I. The mean curvature is infinite and the total curvature finite along $E$.

Condition II. The space coordinates $x^{i}$ of the curves $t=$ const. are functions of s analytic in $\bar{R}$.

Condition III. The curves $C: s=$ const. have an algebraic singularity at $t=0$; that is, within $\bar{R}$ the space coordinates $x^{i}$ of $C$ are analytic in $t^{1 / \alpha}$, where $\alpha$ is an integer called the order of the singularity. $\alpha$ is assumed to be the smallest integer for which this condition is satisfied. $E$ is not a straight line.

8. Analytic structure of the edge of regression. We may now write the equations of the surface in terms of the parametrization $[s, t]$ defined in $\$ 7$. Let $x^{i}=x^{i}(s)$ be the equation of $E$, and let $\xi_{1}^{i}(s), \xi_{2}^{i}(s)$ and $\xi_{3}^{i}(s)$ denote, respectively, the unit tangent, principal normal and binormal vectors of $E$. Then the equations of the surface may be written in the form

$$
X^{i}=x^{i}+a(s, t) \xi_{2}^{i}+b(s, t) \xi_{3}^{i}
$$

where $a(s, t)$ and $b(s, t)$ must, according to Condition II, be analytic in $s$; while according to Condition III, they must be analytic in $t^{1 / \alpha}$. Hence

$$
a=\sum_{1}^{\infty} a_{\mu} \mu^{\mu / \alpha}, \quad b=\sum_{1}^{\infty} b_{\mu} t^{\mu / \alpha}
$$

where $a_{\mu}$ and $b_{\mu}$ are analytic in $s$.

From the definition of the parameter $t$, it follows that $g_{22}=1$, where $g_{\alpha \beta}$ denotes the fundamental tensor in the $[s, t]$ system. We may use this fact to prove that the integer $\alpha$ defined in Condition II (the order of the singularity) is equal to 2 . We have

$$
\frac{\partial X^{i}}{\partial t}=\frac{1}{\alpha}\left[\sum_{1}^{\infty} \mu a_{\mu} \mu^{\mu / \alpha-1}\right] \xi_{2}^{i}+\frac{1}{\alpha}\left[\sum_{1}^{\infty} \mu b_{\mu} \mu^{\mu / \alpha-1}\right] \xi_{3}^{i} .
$$

Hence

$$
g_{22}=\frac{1}{\alpha^{2}} \sum_{\mu=1}^{\infty} \sum_{j=1}^{\mu+2 \alpha-1} j(\mu+2 \alpha-j)\left[a_{j} a_{\mu+2 \alpha-j}+b_{j} b_{\mu+2 \alpha-j}\right] t^{\mu / \alpha}
$$


But since $g_{22}=1$, we have, for $\mu \neq 0$,

$$
\sum_{j=1}^{\mu+2 \alpha-1} j(\mu+2 \alpha-j)\left[a_{j} a_{\mu+2 \alpha-j}+b_{j} b_{\mu+2 \alpha-j}\right]=0 .
$$

We first show that $\alpha \neq 1$. If $\alpha$ were 1 , the coordinates of the surface would be analytic in the parameters $[s, t]$, and the mean curvature would be finite, contrary to Condition I.

Secondly, $\alpha$ cannot be odd. For suppose $\alpha$ were odd and not 1 . We shall prove by induction that

$$
a_{\mu}=b_{\mu}=0 \quad \text { for } \mu \not \equiv 0(\bmod \alpha) .
$$

Equation $(8.2 .2-2 \alpha)$ yields $a_{1}^{2}+b_{1}^{2}=0$ or

$$
a_{1}=b_{1}=0 \text {. }
$$

Suppose we have shown that $a_{\nu}=b_{\nu}=0$ for $\nu \not \equiv 0(\bmod \alpha), \nu<\mu$. Consider equation $(8.2 .2 \mu-2 \alpha)$, where $\mu \neq 0(\bmod \alpha)$. Every one of the products $a_{\lambda} a_{2 \mu-\lambda}, b_{\lambda} b_{2 \mu-\lambda}$ occurring in this equation will involve a term $a_{\nu}$ or $b_{\nu}$ for which $\nu \not \equiv 0(\bmod \alpha)$ and $\nu<\mu$, except the terms $a_{\mu}^{2}+b_{\mu}^{2}$. Hence all the product terms will vanish except $a_{\mu}^{2}+b_{\mu}^{2}$, and equation $(8.2 .2 \mu-2 \alpha)$ will yield $a_{\mu}^{2}+b_{\mu}^{2}=0$, since $2 \mu-2 \alpha \neq 0$. Hence $a_{\mu}=b_{\mu}=0$; and equation (8.3) is established in general, since we have shown independently that $a_{1}=b_{1}=0$. But (8.3) implies that all the terms in the expansions of $a$ and $b$ involving fractional powers of $t$ drop out. Thus $a$ and $b$ would be analytic in $t$, which we have already shown to be inconsistent with Condition I.

Finally, suppose $\alpha$ is even. Then an argument exactly analogous to that of the previous paragraph would enable us to prove that $a_{\mu}=b_{\mu}=0$ for $\mu \neq \equiv$ $(\bmod \alpha / 2)$. Hence $a$ and $b$ would be analytic in $t^{1 / 2}$; and from the minimal property of $\alpha$, as stated in Condition III, we must have $\alpha=2$.

Let us put $\theta=t^{1 / 2}$. Then we have

$$
a=\sum_{\mu=1}^{\infty} a_{\mu} \theta^{\mu}, \quad b=\sum_{\mu=1}^{\infty} b_{\mu} \theta^{\mu} .
$$

The remainder of this section will be occupied with showing that

$$
a_{1}=b_{1}=0, \quad a_{2}=\epsilon= \pm 1, \quad b_{2}=a_{3}=0, \quad b_{3} \neq 0 .
$$

The relations $a_{1}=b_{1}=0$ have already been obtained in (8.4). We shall use the notation $A=o\left(\theta^{n}\right)$ to mean that $\lim _{\theta \rightarrow 0} A / \theta^{n}=k$ where $k$ is a function of $s$ alone. Then we may write (8.1) in the form

$$
X^{i}=x^{i}+\xi_{2}^{i}\left[a_{2} \theta^{2}+a_{3} \theta^{3}+o\left(\theta^{4}\right)\right]+\xi_{3}^{i}\left[b_{2} \theta^{2}+b_{3} \theta^{3}+o\left(\theta^{4}\right)\right] .
$$


From these we obtain, using the Frenet formulas, and denoting by $\rho$ and $\tau$, respectively, the radii of curvature and torsion of $E$,

$$
\begin{aligned}
& g_{11}=1+o\left(\theta^{2}\right), \quad g_{12}=o(\theta), \quad g_{22}=1, \quad g=1+o\left(\theta^{2}\right), \\
& \Omega_{11}=\frac{-b_{2}}{\rho}+o(\theta), \\
& \Omega_{12}=-b_{2}\left(\frac{\partial a_{2}}{\partial s}-\frac{b_{2}}{\tau}\right)+a_{2}\left(\frac{\partial b_{2}}{\partial s}+\frac{a_{2}}{\tau}\right)+o(\theta), \\
& \Omega_{22}=\frac{3}{4 \theta}\left[-a_{3} b_{2}+a_{2} b_{3}+o(\theta)\right] .
\end{aligned}
$$

The mean curvature and the total curvature are thus found to be

$$
\begin{aligned}
K_{m} & =(3 / 4 \theta)\left[-a_{3} b_{2}+a_{2} b_{3}+o(\theta)\right], \\
K & =-\left(3 b_{2} / 4 \rho \theta\right)\left[-a_{3} b_{2}+a_{2} b_{3}+o(\theta)\right] .
\end{aligned}
$$

Since, according to Condition III, $E$ is not a straight line, $1 / \rho \not \equiv 0$. Let us consider the neighborhood of a point at which $1 / \rho \neq 0$. By Condition $I, K_{m}$ is infinite for $\theta=0$, and hence from (8.9)

$$
a_{3} b_{2}-a_{2} b_{3} \neq 0 \text {. }
$$

Again by Condition I, $K$ is finite for $\theta=0$, so that from (8.10) we must have $b_{2}=0$. This fact, together with (8.11), implies that $a_{2} \neq 0, b_{3} \neq 0$. Equation (8.2.0) yields $a_{2}^{2}+b_{2}^{2}=1$, whence $a_{2}=\epsilon= \pm 1$. Finally, from equation (8.2.1) we have

$$
10\left(a_{2} a_{3}+b_{2} b_{3}\right)=0,
$$

so that $a_{3}=0$. This completes the proof of equations (8.6).

In the Cartesian coordinates $\left[y^{1}, y^{2}\right]$, consider the curve $y^{1}=a, y^{2}=b$. In view of (8.6), these equations become

$$
y^{1}=\sum_{2}^{\infty} a_{\mu} \theta^{\mu}, \quad y^{2}=\sum_{3}^{\infty} b_{\mu} \theta^{\mu} .
$$

These are the equations of a curve with a cusp at the origin, of which one branch is the curve $C$, and whose cuspidal tangent coincides with $\xi_{2}^{i}$. Let us choose the sign of $a_{2}$ in such a way that $C$ corresponds to $\theta>0$, and let us denote by $C^{\prime}$ the branch of the curve corresponding to $\theta<0$. As the point $P$ traverses $E$, the curve $C$ will generate the surface $\Sigma$, and the curve $C^{\prime}$ will generate a second surface $\Sigma^{\prime}$ meeting $\Sigma$ cuspidally along $E$. The surfaces $\Sigma$ 
and $\Sigma^{\prime}$, corresponding respectively to $\theta>0$ and $\theta<0$, will be referred to as the two sheets of the edge of regression.

Conversely, if we are given a plane curve whose equations in the $\left[y^{1}, y^{2}\right]$ plane are of the form (8.12), with $a_{2} \neq 0, a_{3}=0, b_{3} \neq 0$, with $a^{i}$ and $b^{i}$ analytic in $s$, and with $\theta^{2}$ equal to the arc length of the curve, measured from the cusp, then the surface

$$
X^{i}=x^{i}+\xi_{2}^{i} \sum_{2}^{\infty} a_{\mu} \theta^{\mu}+\xi_{3}^{i} \sum_{3}^{\infty} b_{\mu} \theta^{\mu}
$$

which is generated by displacing the curve along the analytic curve $E: X^{i}=x^{i}$ in such a way that the point $\theta=0$ lies on $E$ and the cuspidal tangent at $\theta=0$ coincides with the principal normal of $E$, obviously has $E$ as an analytic edge of regression.

9. Local geometrical properties of the edge of regression. This section will be devoted to deriving certain geometrical properties of the edge of regression. Consider a surface defined, in the neighborhood of its edge of regression $E$, by

$$
X^{i}=x^{i}+\xi_{2}^{i} \sum_{2}^{\infty} a_{\mu} \theta^{\mu}+\xi_{3}^{i} \sum_{3}^{\infty} b_{\mu} \theta^{\mu}
$$

where $X^{i}$ is analytic in the half region $\bar{R}:|s|<r_{1}, 0 \leqq \theta^{2}<r_{2}$. The geodesic curvature of $E$ may be defined by

$$
\frac{1}{\rho_{g}}=\frac{-1}{g^{1 / 2}} \frac{\partial g_{11}^{1 / 2}}{\partial t} .
$$

The half region $\bar{R}$ is said to be convex (concave) with respect to its bounding edge $E$ at a point $P$ of $E$ if the geodesic curvature of $E$ is greater than 0 (less than 0 ) at $P$.

We may mention in passing that the concept of convexity as defined above is equivalent to the following more intuitive definition, which may be given a rigid analytical foundation: the half region $\bar{R}$ is convex (concave) with respect to $E$ at $P$ if the geodesics orthogonal to $E$ converge (diverge) in $\bar{R}$ near $P$.

Property I. If $1 / \rho, 1 / \rho_{o}, 1 / \tau$ and $K$ denote, respectively, the curvature, geodesic curvature and torsion of $E$, and the total curvature of the surface along $E$, then

$$
\rho=\epsilon \rho_{g}, \quad K+1 / \tau^{2} \neq 0, \quad \epsilon= \pm 1 .
$$

Proof. From (8.7) we have 


$$
g_{11}=\frac{\partial x^{i}}{\partial s} \frac{\partial x^{i}}{\partial s}=1-2 \frac{a_{2} \theta^{2}}{\rho}+o\left(\theta^{3}\right) .
$$

Hence, from (9.1),

$$
1 / \rho_{o}=a_{2} / \rho+o\left(\theta^{2}\right) .
$$

But since, from (8.6), $a_{2}=\epsilon= \pm 1$, it follows that $\rho=\epsilon \rho_{o}$ along $E$.

Combining equations (8.8) and (8.6), we have

(9.4) $\quad \Omega_{11}=\frac{-3 b_{3}}{2 \rho} \theta+o\left(\theta^{2}\right), \quad \Omega_{12}=\frac{1}{\tau}+o(\theta), \quad \Omega_{22}=\frac{3 a_{2} b_{3}}{4 \theta}[1+o(\theta)]$,

whence $K=-9 a_{2} b_{3}^{2} / 8 \rho-1 / \tau^{2}+o(\theta)$, so that, along $E$,

$$
K+\frac{1}{\tau^{2}}=-\frac{9 a_{2} b_{3}^{2}}{8 \rho}=-\frac{9 b_{3}^{2}}{8 \rho_{o}} \neq 0 .
$$

The case $1 / \rho=1 / \rho_{o}=0$ has already been excluded.

Property II. A surface is convex or concave with respect to its edge of regression according as $K+1 / \tau^{2}<0$ or $>0$ along the edge.

Proof. From the definition of convexity, the surface will be convex or concave with respect to its edge of regression according as $\rho_{g}$ is positive or negative along $E$; that is, because of (9.5), according as $K+1 / \tau^{2}<0$ or $>0$ along E.

PROPERTY III. The edge of regression is the envelope of both sets of asymptotic lines, which are analytic curves near $E$. It is likewise enveloped by one set of lines of curvature, and met orthogonally by the other set.

Proof. The asymptotic lines are given by

$$
\Omega_{11} d s+\left(\Omega_{12} \pm(-g K)^{1 / 2}\right) d t=0 .
$$

We shall consider only the set of asymptotic lines for which the + sign is appropriate; the proof is identical for the other set. Making use of (8.8) and (9.4) we have

$$
\left[\frac{3 b_{3}}{2 \rho} \theta+o\left(\theta^{2}\right)\right] d s+\left[\frac{1}{\tau}+(-K)^{1 / 2}+o(\theta)\right] d t=0,
$$

so that

$$
\frac{d t}{d s}=\frac{3 b_{3} \theta}{2 \rho\left(1 / \tau+(-K)^{1 / 2}\right)}+o\left(\theta^{2}\right) .
$$

This shows that $d t / d s=0$ for $\theta=0$, which implies that the asymptotic lines 
are tangent to $E$. Since the directions of the lines of curvature bisect the angles between the asymptotic lines, it is obvious that one set of lines of curvature will envelope $E$, and the other set will meet it orthogonally.

Putting $d t=2 \theta d \theta$ in (9.6) and inverting, we have

$$
\frac{d s}{d \theta}=\frac{-4 \rho}{3 b_{3}}\left(1 / \tau+(-K)^{1 / 2}\right)+o(\theta) .
$$

Since the right-hand side is analytic in $s$ and $\theta$, it follows that $s$ is analytic in $\theta$. Let $\sigma$ be the arc length along the asymptotic line. Then, recalling that $g_{22}=1$, we have

$$
\left(\frac{d \sigma}{d \theta}\right)^{2}=g_{11}\left(\frac{d s}{d \theta}\right)^{2}+2 g_{12}\left(\frac{d s}{d \theta}\right)+1,
$$

which shows that $d \sigma / d \theta$ is analytic in $\theta$, and hence that $\sigma$ is analytic in $\theta$. But from (9.7), $d \sigma / d \theta$ is obviously not 0 for $\theta=0$, and we conclude that $\theta$ is analytic in $\sigma$, and that the same is true for $s$. Finally, substituting these analytic functions in the equations (8.1) of the surface, we obtain the coordinates of a point on the asymptotic line as analytic functions of its arc length.

We may also show that the asymptotic lines cross over from $\Sigma$ to $\Sigma^{\prime}$ as they envelope $E$. This follows from the fact that, according to equation (9.7), $d \sigma / d \theta \neq 0$ at $E$. Hence, near $E$ a change in the sign of $\sigma$ leads to a change in the sign of $\theta$, and thus to a change from $\Sigma$ to $\Sigma^{\prime}$, or vice versa.

10. The index of a region. Consider an immersed surface whose only singularity is an edge of regression, consisting of one or several pieces. We wish to study the topology of the region bounded by such a singular locus. For this purpose, $\$ 10$ will develop the properties of an index of a region, intimately connected with its Euler characteristic. In $\$ 11$ the index of a region bounded by an edge of regression will be shown to depend upon the indices of the umbilics in that region.

Indices of the type we are considering, and in particular the index of an umbilic, have been studied by Hamburger [9], Blaschke [10], and Franklin [11]. In their treatment of umbilics, these authors depend on the work of Darboux [12] and Gullstrand [13], who investigated the behaviour of the lines of curvature in the neighborhood of an umbilic. The present treatment is quite independent of these earlier discussions, and makes use of the invariants of the surface at an umbilic, rather than the descriptive geometry of the lines of curvature near an umbilic.

We shall now proceed to define and discuss an index $j_{R}$ for a region $R$ of an orientable surface. 
Let $R$ be a region of an orientable surface $\Sigma$, such that the boundary $C$ of $R$ contains no umbilics and has a unique tangent at all except a finite number of points $P_{i}, i=1,2, \cdots$, at each of which it has a corner. Let $C$ be oriented in such a way that the positive tangent vector $T$ of $C$, the normal $N$ of $C$ directed toward $R$, and the normal of $\Sigma$ form a positive triad of directions. Consider the vector $T$ as it traverses $C$ in the positive sense; let $\theta_{i}$ be the angle through which $T$ must rotate in passing from one to the other of the two segments of $C$ which meet at the angle $P$ (the positive sense of rotation is from $T$ to $N$ ). Let $P$ be any point of $C$ and let $V$ be the unit tangent vector to an arbitrarily chosen line of curvature through $P$, with an arbitrary orientation. Finally, let $\theta$ be the positive angle from $V$ to $T$. Then we may define an index $j_{R}$ for the region $R$ by

$$
j_{R}=1-\frac{1}{2 \pi}\left[\oint_{C} d \theta+\sum_{i} \theta_{i}\right] .
$$

Consider the vector $V$ of the above definition, and let $V^{\prime}$ be the tangent vector of the other line of curvature through $P$. It is readily seen that, as $P$ makes a circuit of $C$, the vectors $V$ and $V^{\prime}$ cannot be permuted. For if $1 / \rho_{1}$ and $1 / \rho_{2}$ are the principal curvatures corresponding to $V$ and $V^{\prime}$ respectively, and if $1 / \rho_{1}>1 / \rho_{2}$ at the start, then $1 / \rho_{1}>1 / \rho_{2}$ over the whole path, since it contains no umbilics. This implies that $V$ and $V^{\prime}$ will still correspond to the same principal directions after any circuit.

The vector $V$ may, however, return to its original position with sense reversed. An example illustrating this fact is afforded by a circuit enclosing an umbilic of an ellipsoid, for which the lines of curvature in the neighborhood of an umbilic have the form illustrated in Fig. 1. Consider the region $R$ : $A B C D$, enclosed by two lines of curvature, with right angles at $B$ and $D$. The index is $j_{R}=1-(\pi / 2 \pi)=1 / 2$, and it is clear that the vector $V$ returns: to its original position with sense reversed.

Since $V$ must be carried into $\pm V$ after one circuit, it follows that $\oint_{C} d \theta+\sum_{i} \theta_{i}$ must be some multiple of $\pi$. Hence $j_{R}$ must be some multiple, positive or negative, of $1 / 2$. This has the consequence that the index is unchanged when $R$ is deformed continuously in such a way that its boundary never goes through an umbilic.

THEOREM 10.1. If $R$ is a region which is simply connected and free from umbilics, then $j_{R}=0$.

Proof. Consider any conformal mapping

$$
\Sigma \sim E
$$


which carries the region $R$, the curve $C$, and the vectors $V$ and $T$ of $\Sigma$, respectively, into $\bar{R}, \bar{C}, \bar{V}$ and $\bar{T}$ of the Euclidean plane $E$. Let $D$ be a fixed oriented direction in the plane. Let $\phi$ and $\psi$ denote, respectively, the positive angle from $D$ to $\bar{V}_{L}$ and the positive angle from $D$ to $\bar{T}$. Then $\theta=\psi-\phi$.

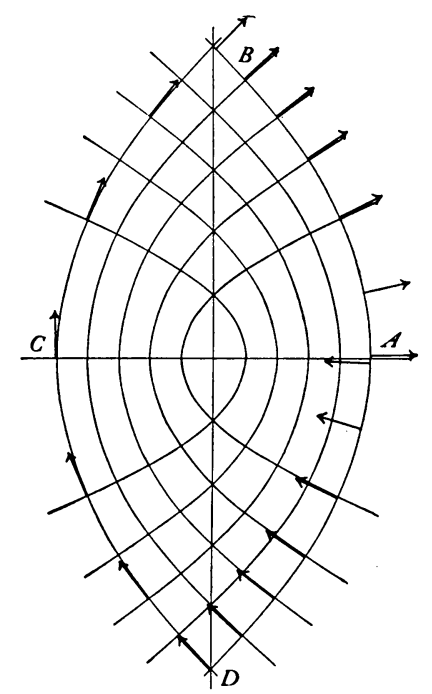

FIG. 1

Since $\bar{R}$ is simply connected, $\bar{C}$ is a simple curve, and the vector $\bar{T}$ will turn through $2 \pi$ in one circuit, which is the total increment in $\psi$ : $\Phi_{\bar{c}} d \psi+\sum_{i} \theta_{i}=2 \pi$. Hence

$$
j_{R}=\frac{1}{2 \pi} \oint_{\bar{c}} d \phi .
$$

Since $R$ is free from umbilics, $V$ is a nonsingular vector field over $R$, and $\bar{V}$ is a nonsingular vector field over $\bar{R}$. Thus the angle $\phi$ is a nonsingular scalar function over $\bar{R}$, and the increment of $\phi$ on traversing the boundary $\bar{C}$ of $\bar{R}$, $\oint_{\bar{c}} d \phi$, equals zero. Hence $j_{R}=0$.

THEOREM 10.2. If $R$ is a region which contains $p$ holes and is free from umbilics,

$$
j_{R}=p=1-\chi(R),
$$

where $\chi(R)$ is the Euler characteristic of $R[14]$.

Proof. We reduce $R$ to a simply connected region $R^{\prime}$ by introducing $p$ cuts, one cut connecting each hole to the outer boundary of $R$. Then $R^{\prime}$ is 
simply connected and free from umbilics, so that $j_{R^{\prime}}=0$. But in making the cuts, we have introduced $4 p$ new angles on the boundary, whose total contribution to $\sum_{i} \theta_{i}$ is $2 p \pi$; so that the index of $R$ has been reduced by $p$. Hence $j_{R}=j_{R^{\prime}}+p=p$.

THEOREM 10.3. Let $R$ be a simply connected region covered by a system of isometric coordinates, so that

$$
g_{11}=g_{22}=\lambda, \quad g_{12}=0
$$

and such that the boundary $C$ of $R$ is free from umbilics and corners. Then

$$
j_{R}=\frac{1}{4 \pi} \oint_{C} d\left(\arcsin \frac{\Omega_{12}}{\lambda N}\right) .
$$

Proof. Let $\alpha$ be the angle from the line $u^{1}=$ const. to the vector $V$ previously defined. Then under the conformal mapping (10.1), the direction $d u^{1}=0$ is carried into a nonsingular vector field over $\bar{R}$. Hence $\phi-\alpha$, the angle from $D$ to $d u^{1}=0$, is a nonsingular scalar function over $\bar{R}$, so that $\oint_{\bar{C}} d(\phi-\alpha)=0$. Thus

$$
j_{R}=\frac{1}{2 \pi} \oint_{\bar{c}} d \phi=\frac{1}{2 \pi} \oint_{\bar{c}} d \alpha
$$

Let us put $a=\Omega_{12}, b=(1 / 2)\left(\Omega_{11}-\Omega_{22}\right)$. Then the lines of curvature are given by

$$
-a d u^{1^{2}}+2 b d u^{1} d u^{2}+a d u^{2^{2}}=0,
$$

from which we find $\sin 2 \alpha=a / \lambda N=\Omega_{12} / \lambda N$, and the theorem is proved.

11. Umbilics. Region bounded by the edge of regression. In this section, Theorem 10.3 will be used to evaluate the index of a region containing an umbilic of the simplest type, in terms of a certain invariant evaluated at the umbilic. The method is perfectly general, however, and could be extended to umbilics of higher order; the present results are intended merely as illustrative. It is then possible to evaluate the Euler characteristic of a region bounded by an edge of regression. We shall assume that the coordinates of the surface in the neighborhood of the umbilic are functions of class $C^{\text {iv }}$ in the parameters. We shall also assume that the parametrization $[u, v]$ is such that the positive tangent to $v=$ const., the positive tangent to $u=$ const., and the surface normal form a positive triad.

We shall deal only with the simplest type of umbilic, namely, those for which

$$
\Gamma=g^{\alpha \beta} \boldsymbol{\varepsilon}^{\gamma \delta} \delta_{\mathcal{\varepsilon}}^{\lambda \mu} \Omega_{\alpha \gamma \lambda} \Omega_{\beta \delta \mu} \neq 0
$$


where $\Omega_{\alpha \beta \gamma}=\Omega_{\alpha \beta, \gamma}$; we have suppressed the comma since $\Omega_{\alpha \beta, \gamma}$ is symmetric in all three indices.

Theorem 11.1. At an umbilic the invariant $\Gamma$ satisfies the identity

$$
\Gamma=\Delta_{2} K-\frac{K_{m}}{2} \Delta_{2} K_{m},
$$

where $K$ and $K_{m}$ are, respectively, the total and mean curvatures.

Proof. In an isometric coordinate system we find that both sides of the identity reduce to

$$
\Gamma=\left(2 / \lambda^{3}\right)\left[\Omega_{111} \Omega_{122}+\Omega_{112} \Omega_{222}-\Omega_{112}^{2}-\Omega_{122}^{2}\right] .
$$

If the simply connected region $R$ contains a single umbilic, then $j_{R}$ is called the index of the umbilic. We shall use the notation sign $(x)$, or sometimes sign $x$, to denote a quantity which is $1,-1$ or 0 according as $x$ is positive, negative or zero.

THEOREM 11.2. If $U$ is an umbilic for which $\Gamma \neq 0$, then its index $j_{U}$ is given by

$$
j_{U}=(1 / 2) \operatorname{sign}(\Gamma)
$$

where $\Gamma$ is given by (11.1).

Proof. Let $R$ be a simply connected region surrounding $U$, covered by an isometric coordinate system $[u, v]$ of which the umbilic is the origin, and bounded by the curve $k$ :

$$
a u+b v=r \sin \beta, \quad c u+d v=r \cos \beta,
$$

where $r$ is constant and

$$
a=\Omega_{112}, \quad b=\Omega_{122}, \quad c=(1 / 2)\left(\Omega_{111}-\Omega_{122}\right), \quad d=(1 / 2)\left(\Omega_{112}-\Omega_{222}\right),
$$

all evaluated at the origin. We shall assume that at the origin $g_{11}=g_{22}=\lambda=1$. Making use of (11.2), it is readily verified that at the origin, $b c-a d=\Gamma / 4$.

Using Taylor's series, we find the following evaluations for $\Omega_{12}$ and $N$ at points of $k$ :

$$
\Omega_{12}=r \sin \beta+o\left(r^{2}\right), \quad N=r+o\left(r^{2}\right) .
$$

Hence, on $k$,

$$
\sin 2 \alpha=\Omega_{12} / \lambda N=\sin \beta+o(r), \quad 2 \alpha=\beta+o(r) .
$$

Consider in the $[u, v]$ plane the conic $H$ defined by (11.3). Since $\Gamma \neq 0$, it is readily shown that $H$ is a nondegenerate ellipse. The curve $k$ will like- 
wise be a nondegenerate closed curve; and as the curve $k$ is traversed in the positive sense, the curve $H$ will also be traversed in the positive sense. That is, the angle $\gamma$ defined by $\gamma=\operatorname{arc} \tan v / u$ will run from 0 to $2 \pi$.

The relationship between $\beta$ and $\gamma$ is given, from (11.3), by

$$
\tan \beta=\frac{a+b \tan \gamma}{c+d \tan \gamma} .
$$

This shows that as $\tan \gamma$ runs from $-\infty$ to $\infty, \tan \beta$ covers the same range in the positive or negative sense. That is, as $\gamma$ increases by $2 \pi, \beta$ increases by $\pm 2 \pi$. From (11.4) we obtain

$$
\frac{d \beta}{d \gamma}=\frac{\Gamma \cos ^{2} \beta \sec ^{2} \gamma}{4(c+d \tan \gamma)^{2}}
$$

which shows that as $\gamma$ increases by $2 \pi, \beta$ increases by $2 \pi$ sign $(\Gamma)$. Hence

$$
j_{U}=\frac{1}{4 \pi} \oint_{k} 2 d \alpha=\frac{1}{4 \pi} \oint_{k} d \beta+o(r)=(1 / 2) \operatorname{sign}(\Gamma)+o(r) .
$$

But $j_{U}$ is independent of the size of the curve $k$, and so $j_{U}=(1 / 2) \operatorname{sign}(\Gamma)$.

THEOREM 11.3. If $R$ is a region entirely bounded by the edge of regression, free from singularities and containing umbilics $U_{i}, i=1,2, \cdots$, with indices $j_{i}$, then the Euler characteristic of $R$ is given by $\chi(R)=\sum_{i} j_{i}$.

Proof. The edge of regression cannot contain umbilics, since along $E$ $K_{m}=\infty$ and $K$ is finite, while at an umbilic $K_{m}^{2}=4 K$. Since the boundary $E$ of $R$ is met orthogonally by one set of lines of curvature, the angle $\theta$ appearing in the definition of $j_{R}$ is constantly equal to $0, \pi / 2, \pi$ or $3 \pi / 2$. Hence by definition, $j_{R}=1$.

Suppose that there are $q$ umbilics, and that $R$ has $p$ holes. Let $R_{i}$, $i=1,2, \cdots, q$, be a set of nonoverlapping simply connected regions, each with a boundary $C_{i}$ which contains neither umbilics nor corners, and such that $U_{i} \subset R_{i}$. Then

$$
j_{R_{i}}=j_{i}=1-\frac{1}{2 \pi} \oint_{C_{i}} d \theta .
$$

On the other hand, the region $R^{\prime}=R-\sum_{i} R_{i}$ is free from umbilics and has $p+q$ holes, and its boundary is $E-\sum_{i} C_{i i}$. Hence

$$
\begin{aligned}
j_{R^{\prime}} & =p+q=1-\left[\oint_{E} d \theta-\sum_{i} \oint_{C_{i}} d \theta\right]=1-\sum_{i}\left(1-j_{R_{i}}\right) \\
& =1+q-\sum_{i} j_{i}
\end{aligned}
$$


so that

$$
\chi(R)=1-p=\sum_{i} j_{i} .
$$

In the case when the umbilics $U_{i}$ are all such that $\Gamma \neq 0$, we may combine Theorems 11.2 and 11.3 to obtain

THEOREM 11.4. If $R$ is a region entirely bounded by the edge of regression, free from singularities, and containing umbilics $U_{i}, i=1,2, \cdots$, such that $\Gamma\left(U_{i}\right) \neq 0$, then the Euler characteristic of $R$ is given by

$$
\chi(R)=\frac{1}{2} \sum_{i} \operatorname{sign} \Gamma\left(U_{i}\right)
$$

It is, of course, always to be understood that $E$ may consist of one or several pieces. As simple illustrations of Theorem 11.3, consider the surfaces of revolution whose meridian curves are shown in Fig. 2. In each case $B C$
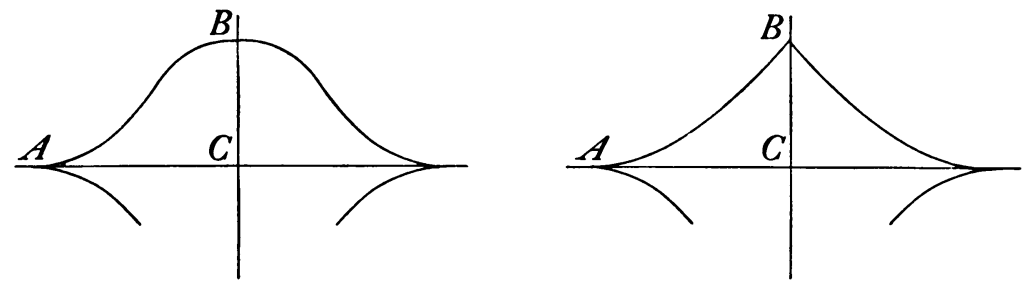

FIG. 2

is the axis of revolution. In the first example, we obtain a simply connected region, free from singularities, and bounded by the edge of regression traced out by the cusp at $A$. For such a region, $\chi(R)=1$; hence Theorem 11.3 implies that there must be one or more umbilics. As a matter of fact, $B$ is an umbilic of index $j_{U}=1$. However, $\Gamma=0$ for that umbilic, so that this illustration does not come under Theorem 11.4. In the second example the region is again simply connected, so that $\chi(R)=1$. Since the surface is of negative curvature, there can be no umbilics. Thus Theorem 11.3 necessitates the existence of a singularity-in this case a conical point at $B$.

\section{Notes AND RefERENCES}

1. W. C. Graustein, Applicability with preservation of both curvatures, Bulletin of the American Mathematical Society, vol. 30 (1924), pp. 19-23; Duke Mathematical Journal, vol. 2 (1936), pp. 177-191.

2. The tensor $\varepsilon^{\alpha \beta}$ is defined and discussed in A. Duschek, Lehrbuch der Differentialgeometrie, Leipzig, Teubner, 1930, p. 99, formula (19).

3. The conditions (5.1) correspond to the conditions " $z=Q / P$ satisfies the equations (4)" of 
Theorem 1 in Graustein's paper in the Duke Mathematical Journal.

4. A. R. Forsyth, Differential Geometry, Cambridge, University Press, 1920, p. 84. (11.14)

5. L. P. Eisenhart, Riemannian Geometry, Princeton, University Press, 1926, p. 30, equation

6. See Theorem 1 in Graustein's paper in the Duke Mathematical Journal.

7. Equation (6.11) is another form of the condition $\Delta_{2} \log (-K)-4 K=0$, which is due to Ricci. See L. Bianchi, Vorlesungen über Differentialgeometrie, translated by M. Lukat, Leipzig, Teubner, 1899, p. 382.

8. Goursat-Hedrick, A Course in Mathematical Analysis, vol. 2, part 2, New York, Ginn, 1917, p. 209.

9. H. Hamburger, Über Kurvenetze mit isolierten singularitäten auf geschlossenen Flächen, Mathematische Zeitschrift, vol. 19 (1924), pp. 50-56.

10. W. Blaschke, Über die Geometrie von Laguerre, IV. Von den Nabelpunkten einer Eifläche, Mathematische Zeitschrift, vol. 24 (1926), pp. 617-621.

11. P. Franklin, Regions of positive and negative curvature on closed surfaces, Journal of Mathematics and Physics, vol. 13 (1934), pp. 253-260.

12. G. Darboux, Théorie des Surfaces, Paris, Gauthier-Villars, 1896, vol. 4, pp. 448-465.

13. A. Gullstrand, Zur Kenntniss der Kreispunkte, Acta Mathematica, vol. 29 (1905), pp. 59-100.

14. See, for example, S. Lefschetz, Topology, American Mathematical Society Colloquium Publications, vol. 12, New York, 1930, p. 44.

LEHIGH UNIVERSITY, Bethlehem, Pa. 